\title{
The chlamydia screening studies: rationale and design
}

\author{
N Low, A McCarthy, J Macleod, C Salisbury, P J Horner, T E Roberts, R Campbell, A Herring, \\ S Skidmore, E Sanford, J A C Sterne, G Davey Smith, A Graham, M Huengsberg, J Ross, M Egger, \\ for the Chlamydia Screening Studies (ClaSS) Group
}

Sex Transm Infect 2004;80:342-348. doi: 10.1136/sti.2003.006197

See end of article for authors' affiliations

.....................

Correspondence to: Dr N Low, Department of Social Medicine, University of Bristol, Canynge Hall, Whiteladies Road, Bristol BS8 2PR, UK; nicola.low@ bristol.ac.uk

Accepted for publication 9 February 2004

\begin{abstract}
Background: Screening has been recommended to reduce the prevalence and morbidity associated with genital chlamydia infection in the United Kingdom.

Methods: We describe the rationale and study design of the Chlamydia Screening Studies (ClaSS), a collaborative project designed to evaluate screening outside genitourinary medicine clinics. A nonselective, active screening approach in 16-39 year olds randomly sampled from 27 general practice lists in the Bristol and Birmingham areas formed the basis of interlinked studies: a case-control study was used to investigate factors to improve the targeting of screening; participants with chlamydia were invited to enrol in a randomised controlled trial to evaluate partner notification conducted in primary care; and laboratory based studies were used to assess the best specimens and tests. We also explored psychosocial effects of screening and partner notification and modelled the cost effectiveness of the programme.

Conclusion: Results from four pilot practices show that mailing of specimens for chlamydia testing is feasible but that it is difficult to achieve high response rates with postal screening. The high prevalence of asymptomatic infection in men suggests that efforts to screen men for chlamydia should be strengthened.
\end{abstract}

S creening efforts can be classified according to the method of recruitment; active or opportunistic, and the population involved; selective or non-selective. Active screening means that people are actively sought and invited for screening. Opportunistic programmes offer screening tests to health service users even if attendances are unrelated to the disease being screened for. Active or opportunistic screening for genital chlamydia infection, the commonest preventable cause of infertility, has been assessed in Sweden, ${ }^{12}$ the United States, ${ }^{34}$ Denmark, $^{56}$ and the Netherlands. ${ }^{78}$ Two randomised controlled trials have found that active screening for chlamydia can reduce the incidence of pelvic inflammatory disease in women by around $50 \% .^{5}$ 9 Opportunistic screening has not been evaluated in a randomised trial but pilot studies in the United Kingdom ${ }^{10}$ and the Netherlands ${ }^{7}$ have shown that this approach is feasible and acceptable and screening activities in Sweden ${ }^{11}$ and the United States ${ }^{3}$ have been associated with reductions in the prevalence of chlamydia and subsequent morbidity.

In 1998 the chief medical officer's expert advisory group on Chlamydia trachomatis recommended action to reduce the prevalence and morbidity associated with chlamydial infection in the United Kingdom, and identified a number of research questions. ${ }^{12}$ The Chlamydia Screening Studies (ClaSS) project was commissioned by the Health Technology Assessment Programme of the National Health Service to address the questions posed by the advisory group. ${ }^{12}$ Here we describe the rationale and study design of this collaborative and multidisciplinary project. We also present preliminary findings from pilot practices where these affected the future conduct of the study. Throughout this paper we use the term chlamydia to mean genital $C$ trachomatis infection.

\section{Rationale and overview of the Chlamydia Screening} Studies (ClaSS) project

The research questions from the expert advisory group included the evaluation of chlamydia screening in non-genitourinary medicine clinic settings, including the best test and specimen to use in men and women, the most effective methods of accessing partners of infected patients, and the most cost effective criteria and appropriate outcomes for targeted screening (box 1 ). ${ }^{12}$ This comprehensive research brief led us to design a series of linked studies, clustered around a large prevalence study in the general population. This paper describes the project as a whole and shows how the design of individual components depend on the core prevalence study. Detailed results from each study component will be reported separately.

Figure 1 gives an overview of the Class project components, and planned study sizes and power calculations are listed in appendix 1 and 2, respectively. The prevalence study used a non-selective, active screening approach in the general population, with participants being asked to send home collected urine and vulval swab specimens for chlamydia testing. This approach enabled us to estimate the population prevalence of chlamydia for use in the economic evaluation, include large numbers of men outside genitourinary clinics and, in laboratory based studies, to evaluate the performance of tests in non-invasive specimens in low prevalence settings. We nested a case-control study within the prevalence survey to investigate risk factors that could be used to improve the targeting of screening, which is relevant to opportunistic as well as active screening programmes, particularly if it identified groups at high risk of chlamydia who do not use health services frequently. Partner notification in the United Kingdom is usually carried out by specialist health advisers based in genitourinary medicine clinics. The workload in a national chlamydia screening programme would exceed the capacity of genitourinary medicine clinics, which may be inaccessible or unacceptable to many people. We designed a randomised controlled partner notification trial to evaluate a partner notification strategy in primary care. Finally, we performed studies of the psychological, emotional, and social effects of the screening and partner notification processes at all stages of the project. We obtained written consent when study packs were sent out for both the prevalence study and, if applicable, for the case-control study. We sought consent 
Box 1 Research questions of the Chlamydia Screening Studies (ClaSS)

Questions posed by the expert advisory group

- How can the cost effectiveness of screening for genital chlamydial infection in non-genitourinary medicine clinic settings be maximised?

- What is the best test and specimen to use for screening for genital chlamydial infection in men and women?

- What are the most effective methods of accessing partners of infected patients for the diagnosis and treatment of genital chlamydial infection?

- What are the most cost effective criteria for targeted screening and which outcomes should be measured?

\section{Additional questions}

- How common is genital chlamydia in men and women in the general population?

- What are the social, emotional, and psychological effects of screening and partner notification for genital chlamydia?

separately for participation in the partner notification trial and in in-depth interviews. For each component, specific objectives and the plan of investigation are described below. Detailed study protocols can be found at www.chlamydia. ac.uk.

\section{Chlamydia prevalence study}

The objectives were to (i) determine the prevalence of chlamydia in a general population sample; (ii) assess the feasibility and acceptability of non-invasive postal chlamydia testing; (iii) identify chlamydia positive cases and negative controls for other components of Class.

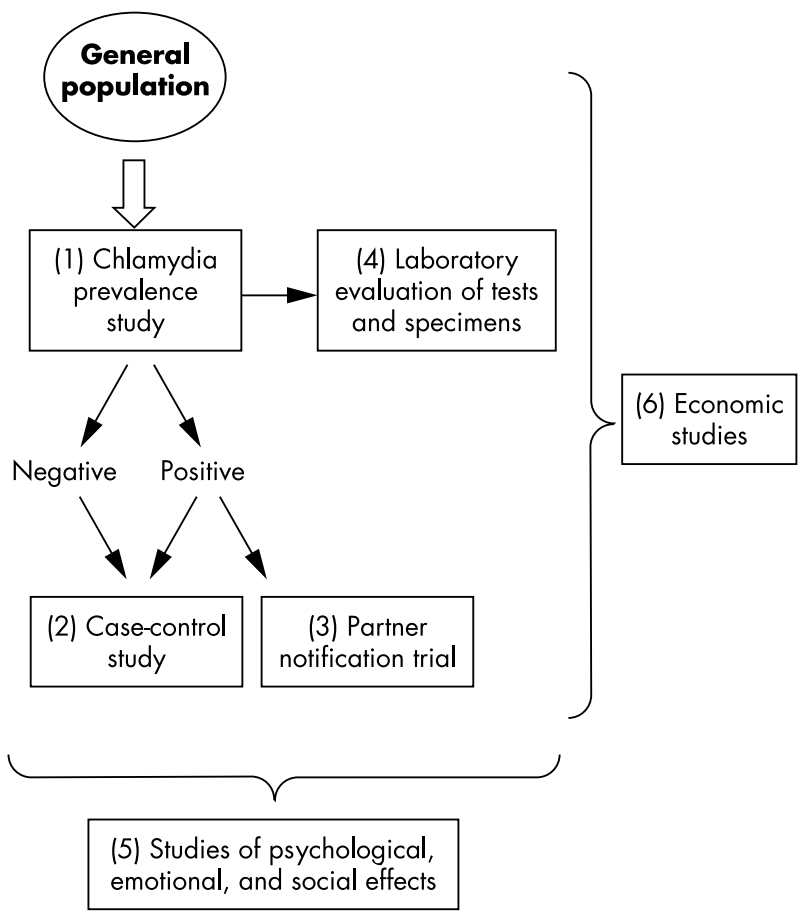

Figure 1 Relation between components of the chlamydia screening studies.
We selected 27 practices from two research networks in the Avon and West Midlands areas to include different practice sizes, urban and rural areas with varying levels of material deprivation, and ethnically heterogeneous populations. Based on the pilot study ${ }^{13}$ we anticipated a response rate of $70 \%$ and assumed that around $20 \%$ of patients in this age group would be "ghost" patients who had moved from the address listed on the GP registers. From a registered total of 85300 1639 year olds we aimed to select 18000 people to achieve a sample size of about 10000 . We sampled at random from a fixed proportion of individuals in each practice, using household as the unit and selecting only one individual per household. We initially selected twice as many 16-25 year olds as 26-39 year olds but after interim analysis in the first four practices (see below) we included only 16-25 year olds to increase the number of chlamydia cases identified. Prevalence estimates were calculated using inverse probability weights to take into account the selection process.

The study pack (box 2) complied with European regulations about mailing of clinical specimens. We explored acceptability and readability of study materials with 11 people chosen at random from those not selected for the prevalence study. We used the first four practices to test methods to optimise the response rate. We randomised participants to receive different types of female swabs (single versus double headed), questionnaires (with or without sexual behaviour questions), mailing of letter from general practitioner (GP) (in advance or with study pack), delivery of study pack (recorded versus standard), and reminders (letter only or additional study pack). In two later practices we examined the effect of a monetary incentive of $£ 10$ given as a gift voucher or donation to a named charity.

We identified "ghost" patients by telephone call or visit to all those who were sent packs but returned neither a specimen nor the form declining participation, and by direct questioning at the time of delivery for courier delivered packs. We sent a reminder letter to all "non-ghosts" not responding within 6 weeks of the first mailing and telephoned those not responding to the second mailing. Finally, we contacted participants by letter once test results were available.

\section{RESULTS FROM PILOT PRACTICES}

Mailing of study packs started on Valentine's day 2001 (14 February). In the first four practices (two in Bristol, two in

\section{Box 2 Contents of the study pack mailed to} potential study participants

- Leaflets about the ClaSS project and a fact sheet about chlamydia

- Invitation letter from the participant's general practice

- Consent form-2 copies

- Brief questionnaire-age, ethnic group, marital status, whether sexually experienced, time since last passed urine, date of last menstrual period for women

- Translation card-to request study materials in Arabic, Bengali, Chinese, Gujerati, Punjabi, Urdu, Vietnamese, Kurdish, Kosovan, Italian, or Somali

- Non-participation form-to decline participation

- Specimen collection materials $-25 \mathrm{ml}$ urine collection pot, dry cotton tipped swab, funnel (for women), plastic glove, instructions, prepaid envelope, packaging materials.

- Pen 
Table 1 Response rates in first four general practices

\begin{tabular}{|c|c|c|c|c|c|c|c|c|c|c|}
\hline \multirow[b]{3}{*}{ Response } & \multicolumn{4}{|l|}{ Men } & \multicolumn{4}{|c|}{ Women } & \multirow{2}{*}{\multicolumn{2}{|c|}{$\frac{\text { Total }}{\text { All ages }}$}} \\
\hline & \multicolumn{2}{|c|}{$16-25$ years } & \multicolumn{2}{|c|}{$26-39$ years } & \multicolumn{2}{|c|}{$16-25$ years } & \multicolumn{2}{|c|}{$26-39$ years } & & \\
\hline & No & (\%) & No & (\%) & No & (\%) & No & (\%) & No & (\%) \\
\hline Responded & 220 & $(28.5)$ & 82 & $(29.7)$ & 310 & (36.7) & 130 & $(42.2)$ & 742 & (34.3) \\
\hline Declined & 219 & $(28.4)$ & 75 & $(27.2)$ & 237 & $(28.0)$ & 99 & (32.1) & 630 & (29.2) \\
\hline Did not respond & 333 & $(43.1)$ & 119 & $(43.1)$ & 258 & $(30.5)$ & 79 & $(25.6)$ & 789 & (36.5) \\
\hline Total & 772 & $(100)$ & 276 & $(100)$ & 805 & $(100)$ & 308 & $(100)$ & 2161 & $(100)$ \\
\hline
\end{tabular}

Birmingham) 2979 study packs were sent. A total of 1842 telephone calls and 1171 visits to participants' homes were made by project staff to establish whether they were resident at the address when the pack was sent. In all, 818 people $(27.4 \%)$ were found to be "ghost patients" and were excluded from the denominator, leaving 2161 potential participants. The proportion of ghost patients was 23\% in women and 32\% in men, and was the same in both age groups. Table 1 shows response rates in the first four practices by age group and sex. The overall response rate was 34\% with 16-25 year old men being least likely and women aged $26-39$ years most likely to provide a specimen.

There were few differences in response rates in the randomised studies, except for a better response when sending a reminder letter rather than another pack to participants not responding to the initial mailing. These results will be reported in detail elsewhere. The roll out of the prevalence study continued while results from the pilot practices were being analysed. The following changes to the mailing procedures were implemented when the analysis was complete: the letter from the GP was sent in advance of the study pack, the questionnaire included questions on sexual behaviour, the female study pack included a double headed swab and we sent reminder letters. We also changed the method of delivery from mail to courier service. Finally, we paid incentives to participants who returned a specimen.

Among participants returning a specimen there were 34 positive results. The overall prevalence of chlamydia was $2.8 \%$ (95\% confidence interval 2.0 to 4.0 ), with similar rates in men and women (table 2). Prevalence was highest in 1625 year olds $(5.0 \%$ in men, $7.2 \%$ in women). There was only one positive test result in the 26-39 year old age group.

\section{Case-control study}

The objective was to identify risk factors for chlamydia that might be used to target screening more effectively. All participants with a positive chlamydia test result were eligible to be cases. For each case the next two consecutive participants with negative results in the same practice and age band (16-25 years or 26-39 years) were selected as controls.

We designed a self completion questionnaire based on the interview used in the National Survey of Sexual Attitudes and Lifestyles. ${ }^{14}$ The interview asked about demographic details, contraceptive use, age at first intercourse, number of sexual partners in the past year, and details of the two most recent sexual partnerships. Without knowing their test result cases and controls were sent the questionnaires and an appointment to attend the surgery. At the surgery the practice nurse received the completed questionnaire and then gave the test result. Participants with negative tests were thanked for their participation. Participants with positive chlamydia test results were treated and then invited to participate in the randomised trial of partner notification strategies. Those with negative tests who had not attended 4 weeks after the date of their initial appointment were sent their result by post. The research health adviser visited participants with positive tests who did not attend to give results and arrange treatment. The data will be analysed using conditional logistic regression to take into account the matched design.

\section{Partner notification trial}

The objective was to determine the feasibility, acceptability, and effectiveness of a simple, nurse led partner notification strategy in GP surgeries compared to referral to a genitourinary medicine clinic for partner notification by a health adviser.

All participants who received their positive chlamydia result at their general practice were eligible. The practice nurse administered azithromycin $\mathrm{l} g$ as a single directly observed dose, or an alternative in the case of allergy or

\begin{tabular}{|c|c|c|c|c|}
\hline Characteristic & No of specimens & Positive & Prev & $(95 \% \mathrm{Cl})$ \\
\hline \multicolumn{5}{|l|}{ Men } \\
\hline $16-25$ years & 219 & 11 & 5.0 & (2.8 to 8.9 ) \\
\hline $26-39$ years & 82 & 0 & 0 & \\
\hline All men* & 301 & 11 & 2.1 & (1.2 to 3.8 ) \\
\hline \multicolumn{5}{|l|}{ Women } \\
\hline $16-25$ years & 309 & 22 & 7.2 & (4.8 to 10.7 ) \\
\hline $26-39$ years & 130 & 1 & 0.8 & (0.1 to 5.2 ) \\
\hline All women* & 439 & 23 & 3.2 & (2.2 to 5.1 ) \\
\hline \multicolumn{5}{|l|}{ Age group } \\
\hline $16-25$ years & 528 & 33 & 6.3 & (4.5 to 8.7 ) \\
\hline $26-39$ years & 212 & 1 & 0.5 & $(0.1$ to 3.2$)$ \\
\hline Total & 740 & 34 & 2.8 & (2.0 to 4.0 ) \\
\hline
\end{tabular}


pregnancy. The practice nurse then informed the patient about the trial and asked for written consent. Allocation was random and concealed using a centralised telephone operated system.

Participants randomised to partner notification in the practice had an interview, designed to last up to 20 minutes, conducted by the practice nurse who issued numbered Class project contact cards for each sexual contact to take to their nearest genitourinary medicine clinic. All practice nurses involved in the study received one and a half days' training about the project and patient led partner notification ${ }^{15}$ using role play with actors and interactive sessions from a research health adviser and GPs. In each centre a research health adviser reinforced key messages at the start of the study in each practice. Participants allocated to the control group were given a card with details of the genitourinary medicine clinic (Milne Centre for Sexual Health in Bristol, Whittall Street Clinic in Birmingham) and asked to make an appointment with the research health adviser. The research health adviser conducted partner notification by patient led, provider led, or contract referral, ${ }^{15}$ using their own judgment and issued contact cards. Participants declining randomisation were treated according to their preferred method for partner notification.

The main outcome measure was originally defined as antibiotic treatment of sexual partners, which was confirmed by return of ClaSS project contact cards to the coordinating centre. When it became clear that few contact cards were being returned we made a protocol change to specify a combined primary outcome that also included evidence of partner attendance at a genitourinary medicine clinic and verbal report by the index case that the partner had received treatment (see www.chlamydia.ac.uk/partnernotif.htm). Secondary outcomes included number of sexual partners elicited in the sexual history; proportion of index cases randomised to genitourinary clinic arm who presented at the clinic for partner notification; chlamydia positivity rate at 6 weeks.

\section{Laboratory evaluation of tests and specimens}

Objectives included (i) determination of the most appropriate non-invasive female specimen; (ii) comparison of nucleic acid amplification tests with an amplified enzyme linked immunoassay on non-invasive specimens; (iii) comparison of performance characteristics, stability, and ease of use of two nucleic acid amplification systems using non-invasive specimens; (iv) determination of the efficacy of specimen pooling in a low prevalence population.

All participants were asked to provide $25 \mathrm{ml}$ of early morning first void urine. Women were also asked to collect a non-invasive vulval swab consisting of two cotton tipped swabs on a split $7 \mathrm{~cm}$ plastic shaft, with one swab being used for each assay. Specimens were mailed to the Public Health Laboratory Service laboratories in each site and tested on arrival according to standard operating procedures. We compared sensitivity and specificity, stability, labour requirements, use of laboratory space, and costs for urine and vulval specimens as substrates for nucleic acid amplification tests. Determining the most appropriate test for women also included an assessment of acceptability using results from the qualitative studies and response rates obtained when women were randomised to receiving either a single or double headed swab. We evaluated the Cobas Amplicor CT polymerase chain reaction test (Roche Diagnostics, Basel, Switzerland) in Bristol, BD Probe Tec ET strand displacement amplification test (Becton Dickinson, Franklin Lakes, NJ, USA) in Birmingham and IDEIA PCE enzyme linked immunoassay (Dako, Copenhagen, Denmark) in both Bristol and Birmingham. Strand displacement amplification and enzyme immunoassay tests could not be compared directly with urine because of volume requirements. Samples with results that were equivocal or in the negative grey zone by IDEIA were retested; otherwise the residual specimen was frozen and stored at $-20^{\circ} \mathrm{C}$. All samples giving initially positive results were confirmed according to a testing algorithm requiring repeat testing (see www.chlamydia.ac. uk/evaldiag.htm). All samples confirmed as positive were regarded as true positives.

Coded panels of specimens, consisting of all positive or discordant specimens and a random sample of two negative specimens for each positive, were exchanged between the two centres for retesting. We assessed specimen stability at ambient temperatures in women attending the Milne Centre in Bristol using $25 \mathrm{ml}$ of first catch urine and a physician collected vulval specimen, in addition to the normal endocervical and urethral swabs. The additional specimens from women with positive IDEIA results were left at ambient temperature for 24 or 48 hours before freezing at $-20^{\circ} \mathrm{C}$ and batch testing by Cobas polymerase chain reaction.

We determined the performance of nucleic acid amplification tests on pool sizes of four and eight samples. Dilution studies were performed before pooling to assess the sensitivity of the assays in detecting target material in up to eightfold dilution.

\section{Social research}

We used qualitative and quantitative methods to (i) assess the acceptability and psychological and social effects of screening for chlamydia by home testing; (ii) providing partner notification in primary care; and (iii) to elicit the views and experiences of primary care staff.

We used in-depth interviews to examine the feelings and experiences of study participants and health professionals. Interviews were conducted in a setting convenient to the interviewee and were audiotaped and fully transcribed. Interviewees were purposively selected to ensure a balanced sample of men and women from different age groups. We interviewed participants with positive or negative results in the prevalence study. The check list of topics included what the term chlamydia meant; feelings about being tested for a sexually transmitted infection and about providing urine and vulval specimens; expectations about test results and reactions to receiving the results; effects on personal relationships. We also interviewed people who declined testing to explore reasons why people did not want to be screened. Interviewees with positive chlamydia results were asked about their experience of partner notification by a practice nurse or health adviser at a genitourinary medicine clinic. Interviews with health professionals focused on experiences and views of managing patients found through screening to have chlamydia.

We used two measures of anxiety and wellbeing, the Hospital Anxiety and Depression scale ${ }^{16}$ and Rosenberg SelfEsteem scale, ${ }^{17}$ to monitor changes in these outcomes during screening. We mailed questionnaires, with a letter of invitation from the participant's general practice to a proportion of participants in each practice 4 weeks before the study pack was sent out. Repeat questionnaires were then sent to the same participants after receiving the study pack and after receiving a negative result.

\section{Economic studies}

The objectives were to determine the relative cost effectiveness of (i) population screening for chlamydia compared to the current strategy of routine screening only in genitourinary medicine clinics; (ii) the two partner notification strategies; (iii) the different laboratory tests. 
The evaluation was carried out from a societal perspective based on "major outcome averted," defined as the occurrence of at least one of: pelvic inflammatory disease, ectopic pregnancy, and infertility. We used a modelling approach to estimate cost effectiveness because of the time lag between implementation and any future benefits of chlamydia screening, and the number of possible scenarios. We chose to use discrete event simulation using individual level data obtained, where possible, from the Class project. This approach addressed the requirements for a dynamic modelling strategy that considers the complexities of changes in the prevalence of infection as a result of the screening programme and the impact of re-infection. ${ }^{12}{ }^{18}$ It allows comparisons of the effectiveness of different screening approaches and the identification of indicators, such as prevalence, incidence, and re-infection rates, that can be used to measure the impact of chlamydia screening.

Primary data collected within the Class project included cost and resource use data about; study packs and delivery, laboratory tests, treatment, staff training, health adviser time and private costs to individuals as a result of the screening programme. Epidemiological and resource use data on long term outcomes associated with chlamydia were collected from published and unpublished sources and expert opinion.

\section{DISCUSSION}

The ClaSS project is a series of linked studies designed to answer key questions about screening for chlamydia that incorporates a range of methodology from epidemiology, qualitative, economic and laboratory research. Experience from the first four general practices demonstrated that the project was feasible. The response to a mailed request for a home collected specimen for chlamydia testing was, however, only $34 \%$. Among those returning a specimen the prevalence of chlamydia in men was similar to that in women and was very low over the age of 25 years. Of note, the inclusion of questions about recent sexual partnerships did not prejudice the response rate.

\section{Strengths and weaknesses}

Strengths of ClaSS include its size, population based design, inclusion of men, location in primary care and the integral collection of clinical epidemiological, laboratory, psychosocial, and economic data. Chlamydia is largely asymptomatic so knowing the population prevalence is essential for estimating the burden of the disease, its complications and transmission dynamics, and modelling the effectiveness and cost effectiveness of screening. A population based study is also important because the tests for chlamydia have been developed for use in clinical settings. The performance characteristics may well differ when used as screening tests in a low prevalence asymptomatic population with lower chlamydial load. By including men we are obtaining information about a population that has been largely excluded from screening efforts, including the Department of Health sponsored pilot studies of opportunistic screening, ${ }^{10}{ }^{19}$ but whose importance in chlamydia prevention is increasingly being recognised..$^{20}$

By using home collected specimens and managing diagnosed infections in primary care we avoided much of the increased workload associated with opportunistic testing. We can also examine the processes and implications in what will be a major setting if chlamydia screening is fully implemented in the United Kingdom. General practices already account for over half of chlamydia specimens and a third of positive results in some areas (Wendi Slater, Avon System for Surveillance of Sexually Transmitted Infections, personal communication). As activity increases further the role of primary care in partner notification will also increase. The few randomised trials of partner notification ${ }^{21}$ were based in specialist clinics. Our trial will provide essential data on the effectiveness of practice nurses who have received brief training in sexual history taking and partner notification and continuing support from health advisers.

The response rate achieved in postal surveys is important for both internal and external validity. Unfortunately, it was lower in the first four practices than expected from our pilot study $^{13}$ and the reasons for this are currently unclear. Analysis of in-depth interviews with non-responders will provide some insight into why people chose not to participate. Our response rate was, however, consistent with other population based studies, which were published after this study was designed. ${ }^{522}$ A sustained media campaign, which would accompany a national screening programme could increase response rates substantially. Non-response can introduce participation bias that could distort the estimates of chlamydia prevalence. We will be able to determine the likelihood of bias if participation was related to sexual behaviour by comparing our study population's sexual experience and numbers of overall and new sexual partners with nationally representative information. ${ }^{14}$ One consequence of the response rate achieved is that the sample size of other study components that rely on participants with positive chlamydia results will also be smaller.

\section{Chlamydia prevalence}

The initial estimates of chlamydia prevalence in this 1639 year old population $(2.1 \%$ in men and $3.2 \%$ in women) were broadly consistent with other population based surveys. The British National Survey of Sexual Attitudes and Lifestyles 2000 found chlamydia in $2.2 \%$ of men and $1.5 \%$ of women aged 16-44 years. ${ }^{23}$ A study in Amsterdam that also sampled from general practice lists found a prevalence of $2.3 \%$ in men and $2.9 \%$ in women among 4560 15-40 year olds. ${ }^{8}$ Opportunistic screening in Sweden, largely targeted at women, was associated with a reduction in the prevalence of infection until 1996. A possible contributing factor to the increase since then is that this approach has reached its limit and control of this infection will require screening in men as well. $^{24}$ The finding that the prevalence of undiagnosed chlamydia is similar in men and women fills an important gap in knowledge that should influence decisions about the benefits of including men in chlamydia screening programmes. ${ }^{25}$

\section{CONCLUSIONS}

The results from our pilot practices show that mailing of specimens for chlamydia testing is feasible but that it is difficult to achieve high response rates with postal screening. The high prevalence of asymptomatic infection in men suggests that efforts to screen men for chlamydia should be strengthened.

\section{CONTRIBUTORS}

NL drafted the manuscript; ME was the principal investigator of ClaSS; AMCC managed the project; ES and JS did the statistical analysis; ME, NL, JM, CS, PH, RC, TR, AH, SS, GDS, JS, AG, JR, and $\mathrm{MH}$ contributed to study design and/or supervision; all authors helped revise the manuscript.

\section{Authors' affiliations}

N Low, A McCarthy, R Campbell, E Sanford, J A C Sterne,

G Davey Smith, M Egger, Department of Social Medicine, University of Bristol, Bristol BS8 2PR, UK

J Macleod, Department of Primary Care, University of Birmingham, Birmingham B15 2TT, UK 
C Salisbury, A Graham, Primary Health Care, University of Bristol, Bristol BS6 6JL, UK

P J Horner, The Milne Centre, United Bristol Healthcare Trust, Bristol BS2 $8 \mathrm{HW}, \mathrm{UK}$

A Herring, Public Health Laboratory Service Bristol Laboratory, Bristol BS2 8EL, UK
S Skidmore, Public Health Laboratory Service Birmingham Laboratory, Birmingham B9 5ST, UK

T E Roberts, Health Services Management Centre, University of Birmingham B15 2RT, UK

M Huengsberg, J Ross, Whittall Street Clinic, Heart of Birmingham

Primary Care Trust, Birmingham B4 6DH, UK

\section{APPENDIX 1}

Table A1.1 Chlamydia screening studies: summary of planned sample sizes

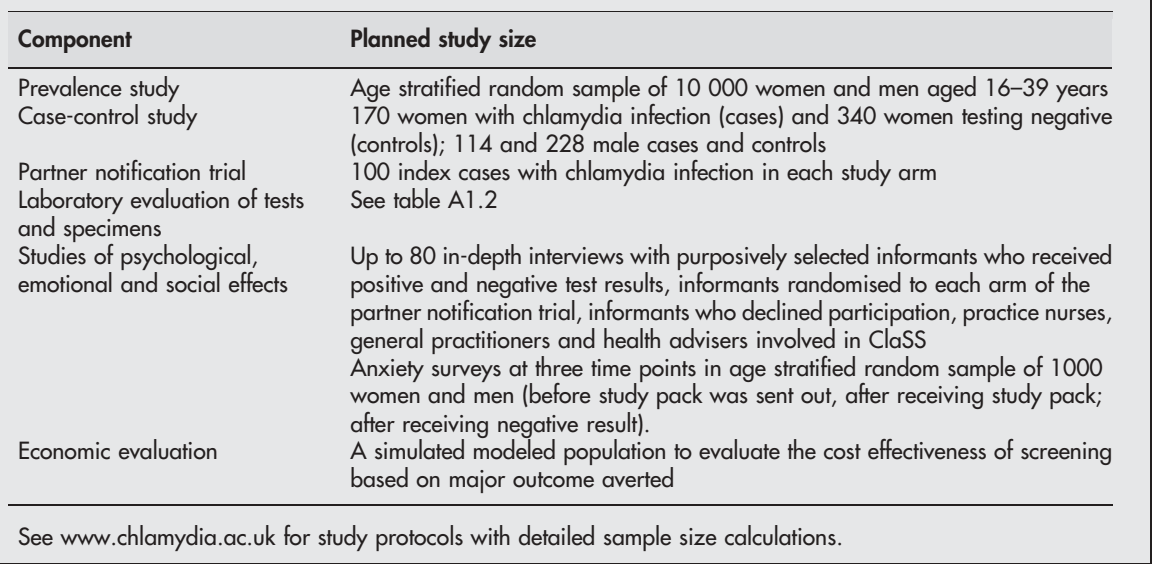

Table A1.2 Summary and planned sample size of laboratory studies

\begin{tabular}{|c|c|c|c|}
\hline Objective & Centre & $\begin{array}{l}\text { Specimens (planned } \\
\text { sample size) }\end{array}$ & Tests \\
\hline \multirow[t]{2}{*}{$\begin{array}{l}\text { Comparison of female } \\
\text { specimens }\end{array}$} & Bristol & $\begin{array}{l}\text { Female urine } v \text { vulval swabs } \\
(3000)\end{array}$ & PCR \\
\hline & Birmingham & $\begin{array}{l}\text { Female urine } v \text { vulval swabs } \\
(3000)\end{array}$ & SDA \\
\hline \multirow[t]{2}{*}{ Test comparison 1} & Bristol & $\begin{array}{l}\text { Male urine, vulval swabs } \\
(2000,3000)\end{array}$ & PCR $v$ EIA \\
\hline & Birmingham & Vulval swabs $(3000)$ & SDA $v$ EIA \\
\hline $\begin{array}{l}\text { Test comparison } 2 \\
\text { Quality assurance }\end{array}$ & $\begin{array}{l}\text { Exchange of specimens } \\
\text { between Bristol and } \\
\text { Birmingham }\end{array}$ & $\begin{array}{l}\text { Male urine, vulval swabs } \\
(4000,6000)\end{array}$ & PCR $\vee$ SDA \\
\hline $\begin{array}{l}\text { Examine specimen } \\
\text { stability }\end{array}$ & Bristol & $\begin{array}{l}\text { Female urine, vulval swabs from } \\
\text { genitourinary clinic ( } 300 \text { each) }\end{array}$ & PCR \\
\hline $\begin{array}{l}\text { Determine optimum } \\
\text { pool size }\end{array}$ & Bristol & $\begin{array}{l}\text { Male urine, vulval swabs } \\
(100,200)\end{array}$ & $\begin{array}{l}\text { PCR, pool sizes of } \\
4 \text { and } 8\end{array}$ \\
\hline
\end{tabular}

\section{APPENDIX 2}

\section{POWER CALCULATIONS FOR CLASS COMPONENT STUDIES}

Further details can be found at www.chlamydia.ac.uk/ arms.htm.

\section{Prevalence study}

We estimated that the prevalence of chlamydial infection would be between $4 \%$ and $6 \%$ in the younger age group and $0.5 \%$ to $2 \%$ in the older age group. The numbers of men and women in each age group assumed that response rates would be higher in women than men such that women would comprise two thirds of the study population in each age group. The intracluster correlation coefficient is unknown.
We assumed intracluster correlation coefficients of 0.001 and 0.002 . These result in design factors of 1.14 and 1.26 for women and 1.10 and 1.18 for men.

\section{Case-control study}

The number of cases required assumes a higher response rate in women $(60 \%)$ than men $(40 \%)$. The number of cases was calculated using the estimated prevalences above and the oversampling of cases from the younger age group.

\section{Partner notification study}

The expected success rates were based on a pilot study at the Milne Centre for Sexual Health, Bristol. The comparison is based on the intention to undertake partner notification and took into account the fact that a proportion of patients referred from primary care to genitourinary clinics would not attend. 
Table A2.1 Precision of hypothetical prevalence estimates at differing intraclass correlation coefficients

\begin{tabular}{|c|c|c|c|c|c|c|}
\hline \multirow[b]{3}{*}{ ICC } & \multicolumn{3}{|c|}{ Age group $16-25$ years } & \multicolumn{3}{|c|}{ Age group $26-39$ years } \\
\hline & \multirow[b]{2}{*}{ Prevalence } & Women (if $n=4000$ ) & Men (if $n=2666$ ) & \multirow[b]{2}{*}{ Prevalence } & \multirow{2}{*}{$\begin{array}{l}\text { Women (if } n=2000 \text { ) } \\
95 \% \mathrm{Cl}\end{array}$} & \multirow[t]{2}{*}{ Men lif $n=1333$} \\
\hline & & $95 \% \mathrm{Cl}$ & & & & \\
\hline 0.001 & $4.0 \%$ & 3.31 to 4.69 & 3.18 to 4.82 & $0.5 \%$ & 0.15 to 0.85 & 0.07 to 0.93 \\
\hline 0.002 & $4.0 \%$ & 3.23 to 4.77 & 3.12 to 4.88 & $0.5 \%$ & 0.11 to 0.89 & 0.04 to 0.96 \\
\hline 0.001 & $6.0 \%$ & 5.16 to 6.84 & 5.00 to 6.99 & $2.0 \%$ & 1.30 to 2.70 & 1.18 to 2.82 \\
\hline 0.002 & $6.0 \%$ & 5.07 to 6.93 & 4.94 to 7.06 & $2.0 \%$ & 1.23 to 2.77 & 1.12 to 2.88 \\
\hline
\end{tabular}

ICC, intraclass correlation coefficient. Binomial confidence intervals are shown.

Table A2.2 Number of female and male chlamydia cases needed according to desired odds ratio and study power

\begin{tabular}{|c|c|c|c|c|c|c|c|}
\hline \multicolumn{4}{|l|}{ Women } & \multicolumn{4}{|l|}{ Men } \\
\hline \multirow[b]{2}{*}{ No of cases } & \multicolumn{3}{|c|}{ Detectable odds ratio } & \multirow[b]{2}{*}{ No of cases } & \multicolumn{3}{|c|}{ Detectable odds ratio } \\
\hline & Powe & & & & Powe & & \\
\hline & $80 \%$ & $90 \%$ & $95 \%$ & & $80 \%$ & $90 \%$ & $95 \%$ \\
\hline 170 & 1.89 & 2.06 & 2.20 & 114 & 2.16 & 2.39 & 2.59 \\
\hline 220 & 1.75 & 1.89 & 2.00 & 146 & 1.98 & 2.18 & 2.33 \\
\hline 280 & 1.65 & 1.76 & 1.86 & 197 & 1.81 & 1.96 & 2.06 \\
\hline
\end{tabular}

Calculations based on a two sided significance level of 0.05 in univariate analysis ignoring matching. Frequency of exposure in controls $20 \%$.

Table A2.3 Number of patients in each study arm required according to desired odds ratio and study power

\begin{tabular}{|c|c|c|c|c|c|}
\hline \multirow{2}{*}{$\begin{array}{l}\text { Success rate in GU } \\
\text { clinic }^{*}\end{array}$} & \multirow{2}{*}{$\begin{array}{l}\text { Success rate in GP } \\
\text { surgeries* }^{*}\end{array}$} & \multirow[b]{2}{*}{ Difference } & \multicolumn{3}{|c|}{ No of index patients in each study arm } \\
\hline & & & $80 \%$ Power & $90 \%$ Power & $95 \%$ Power \\
\hline $40 \%$ & $30 \%$ & $-10 \%$ & 376 & 496 & 609 \\
\hline $40 \%$ & $20 \%$ & $-20 \%$ & 91 & 119 & 144 \\
\hline $50 \%$ & $40 \%$ & $-10 \%$ & 408 & 538 & 661 \\
\hline $50 \%$ & $30 \%$ & $-20 \%$ & 103 & 134 & 163 \\
\hline $60 \%$ & $50 \%$ & $-10 \%$ & 408 & 538 & 538 \\
\hline $60 \%$ & $40 \%$ & $-20 \%$ & 107 & 140 & 170 \\
\hline
\end{tabular}

*Proportion of index patients with one or more partners who received treatment, defined as return of a study contact slip, matching of name on clinic computer or report by index case at telephone follow up. Based on a continuity corrected $\chi^{2}$ test with a 0.05 two sided significance level.

\section{REFERENCES}

1 Herrmann B, Egger M. Genital Chlamydia trachomatis infections in Uppsala County, Sweden, 1985-1993: declining rates for how much longer? Sex Transm Dis 1995;22:253-60.

2 Kamwendo F, Forslin L, Bodin L, et al. Programmes to reduce pelvic inflamm atory disease-the Swedish experience. Lancet 1998;351(Suppl 3):25-8.

3 Hillis SD, Nakashima A, Amsterdam L, et al. The impact of a comprehensive chlamydia prevention program in Wisconsin. Fam Plann Perspect 1995;27:108-11

4 Addiss D, Vaughin ML, Ludka D, et al. Decreased prevalence of Chlamydia trachomatis infection associated with a selective screening program in family planning clinics in Wisconsin. Sex Transm Dis 1993;20:28-34.

5 Østergaard L, Andersen B, Olesen F, et al. Efficacy of home sampling for screening of Chlamydia trachomatis: randomised study. BMJ 1998:317:26-7.

6 Østergaard L, Andersen B, Møller JK, et al. Home sampling versus conventional swab sampling for screening of chlamydia trachomatis in women: a cluster-randomized 1-year follow-up study. Clin Infect Dis 2000;31:951-7.

7 Van den Hoek JAR, Mulder-Folkerts DKF, Coutinho RA, et al. Opportunistic screening for genital infections with Chlamydia trachomatis among the sexually active population of Amsterdam, I. Over $90 \%$ participation and almost $5 \%$ prevalence. Nederlands Tijdschrift voor Geneeskunde 1999;143:668-72.

8 Van Valkengoed GM, Boeke AJP, Van den Brule AJC, et al. Systematic screening for asymptomatic Chlamydia trachomatis infections by home obtained mailed urine samples in men and women in general practice. Nederlands Tijdschrift voor Geneeskunde 1999;143:672-6.

9 Scholes D, Stergachis A, Heidrich FE, et al. Prevention of pelvic inflammatory disease by screening for cervical chlamydial infection. N Engl J Med 1996:334:1362-6.

10 Pimenta JM, Catchpole M, Rogers PA, et al. Opportunistic screening for genital chlamydial infection. I: Acceptability of urine testing in primary and secondary healthcare settings. Sex Transm Infect 2003;79:16-21.

11 Egger M, Low N, Davey Smith G, et al. Screening for chlamydial infections and the risk of ectopic pregnancy in a county in Sweden: ecological analysis. BMJ 1998;316:1776-80.
12 Department of Health. CMO's expert advisory group on Chlamydia trachomatis. London: $\mathrm{DoH}, 1998$.

13 Macleod J, Rowsell R, Horner P, et al. Postal urine specimens: Are they a feasible method for genital chlamydial infection screening? Br J Gen Pract 1999;49:455-8.

14 Johnson AM, Mercer CH, Erens B, et al. Sexual behaviour in Britain: partnerships, practices and HIV risk behaviours. Lancet 2001;358:1835-42.

15 Society of Sexual Health Advisers. The manual for sexual health advisers. www.ssha.info/public/manual/ha_manual_2004_section_a.pdf, accessed 11 June 2004.

16 Zigmond AS, Snaith RP. The Hospital Anxiety and Depression Scale. Acta Psychiatr Scand 1983;67:361-70.

17 Rosenberg M. Society and the adolescent self-image. Middletown, CT: Wesleyan University Press, 1989.

18 Kretzschmar M, Van Duynhoven YTHP, Severijnen AJ. Modeling prevention strategies for gonorrhea and chlamydia using stochastic network simulations. Am J Epidemiol 1996; 144:306-17

19 Pimenta JM, Catchpole M, Rogers PA, et al. Opportunistic screening for genital chlamydial infection. II: Prevalence among healthcare attenders, outcome, and evaluation of positive cases. Sex Transm Infect 2003:79:22-7.

20 Fenton KA. Screening men for Chlamydia trachomatis infection: have we fully explored the possibilities? Commun Dis Public Health 2000;3:86-9.

21 Mathews C, Coetzee N, Zwarenstein M, et al. Strategies for partner notification for sexually transmitted diseases. Cochrane Database Syst Rev 2001:CD002843.

22 Stephenson J, Carder C, Copas A, et al. Home screening for chlamydial genital infection: is it acceptable to young men and women? Sex Transm Infect 2000;76:25-7.

23 Fenton KA, Korovessis C, Johnson AM, et al. Sexual behaviour in Britain reported sexually transmitted infections and prevalent genital Chlamydia trachomatis infection. Lancet $2001 ; 358: 1851-4$.

24 Low N, Egger M. What should we do about screening for genital chlamydia? Int J Epidemiol 2002;31:891-3.

25 Duncan B, Hart G. Sexuality and health: the hidden costs of screening for Chlamydia trachomatis. BMJ 1999;318:931-3. 